\title{
« Les criminologues n'ont jamais rien fait à propos du problème du crime »
}

Entretien de Howard S. Becker avec Didier Bigo, Laurent Bonelli et Fabienne Brion ( $1^{\text {er }}$ octobre 2012)

Howard S. Becker

\section{OpenEdition}

Journals

Édition électronique

URL : http://journals.openedition.org/conflits/18880

DOI : $10.4000 /$ conflits. 18880

ISSN : $1777-5345$

Éditeur :

CCLS - Centre d'études sur les conflits lilberté et sécurité, L'Harmattan

Édition imprimée

Date de publication : 31 décembre 2014

Pagination : 27-41

ISBN : 978-2-343-05760-6

ISSN : 1157-996X

Référence électronique

Howard S. Becker, " «Les criminologues n'ont jamais rien fait à propos du problème du crime » », Cultures \& Conflits [En ligne], 94-95-96 | été-automne-hiver 2014, mis en ligne le 20 février 2016, consulté le 30 mars 2021. URL : http://journals.openedition.org/conflits/18880 ; DOI : https://doi.org/ $10.4000 /$ conflits. 18880 


\title{
«Les criminologues n'ont jamais rien fait à propos du problème du crime »
}

\author{
Entretien de Howard S. Becker avec Didier Bigo, Laurent \\ Bonelli et Fabienne Brion (1 ${ }^{\text {er }}$ octobre 2012)
}

Cultures E Conflits (CEC): Vous êtes considéré comme l'un des pères de la sociologie interactionniste de la déviance et on aimerait savoir comment vous avez croisé la criminologie dans votre vie intellectuelle, c'est-à-dire comment, par exemple, les criminologues américains ont-ils reçu vos travaux. Quel type de commentaires, de critiques ont-ils formulés? Et qu'avez-vous à leur répondre?

Howard S. Becker (H.B.) : La situation de la criminologie aux États-Unis diffère quelque peu, je pense, de ce qu'elle est en France. Il y a toujours eu, pourrait-on dire, deux façons d'approcher ces questions; deux façons qui au départ étaient un peu mêlées, mais se sont séparées.

La première se caractérise par sa prétention à « l'amélioration » : « Aidons la société à se libérer de ces problèmes ». L'idée était de découvrir qui sont les criminels et d'apprendre comment les trouver et s'en débarrasser. Vous connaissez ces tentatives de découvrir comment la forme de votre tête peut indiquer des tendances criminelles ${ }^{1}$.

La seconde est en quelque sorte aux racines du type de travail que $j$ 'ai mené. Beaucoup plus sociologique, elle relève davantage d'une science abstraite ou généralisante, qui ne considère pas tout ou presque tout comme allant de soi.

1. Howard Becker renvoie aux thèses de l'anatomiste Franz Joseph Gall - inventeur de la "phrénologie » - et aux applications criminologiques auxquelles elles ont donné lieu à la fin du XIXe siècle, qu'il s'agisse des travaux de Cesare Lombroso - l'auteur de L'nomo delinquente (1876) et le fondateur de l'anthropologie criminelle - ou de ceux d'Alphonse Bertillon - l'inventeur de l'anthopométrie judiciaire et le premier directeur du Service de l'Identité judiciaire. Sur la généalogie de cette problématisation, voir, entre autres références, Ducros A., « Phrénologie, Criminologie, Anthropologie : une interrogation continue sur anatomie et comportement ", Bulletins et Mémoires de la Société d'anthropologie de Paris, Nouvelle Série, tome 10, fascicule 3-4, 1998, pp. 471-476 ; et Labadie J.-M., " Corps et crime. De Lavater (1775) à Lombroso (1876) », in Debuyst C., Digneffe F., Labadie J.-M., Pires A., Histoire des savoirs sur le crime et la peine, vol. 1, Bruxelles, Larcier, 1995, pp. 315-373. 
Bien sûr, je n'ai jamais été criminologue : enseigner comme criminologue, même à l'intérieur de la sociologie, signifiait s'impliquer dans le système de justice criminelle (puisque désormais ce sont ces mots - « justice criminelle »qui sont utilisés). Certes, la pression à se débarrasser de la délinquance était forte. Mais il n’y avait pas que cela : la sociologie américaine des premières années insistait avec force sur l'observation rapprochée et détaillée de choses supposément criminelles. Ainsi, il y a le grand ouvrage de Frederic Thrasher, The Gang: A Study of 1313 Gangs in Chicago, (Chicago, University of Chicago Press, 1927) - j'ai toujours adoré la précision du nombre, 1313 , vraiment ? (rires). Le cadre, c'était vraiment la vision de la ville qu'avait Robert Park. Park était un élève de Simmel (de sorte que mon lignage, c'est Simmel, Park et Everett Hughes). C'était aussi un ancien journaliste professionnel. Son approche, était : «Oh, regarde ça ! Comme c'est intéressant ! » Il a écrit un essai formidable sur la ville comme laboratoire du comportement humain ; c'est une façon de penser ce type de criminologie.

Edwin Sutherland - un criminologue, mais pas de la sorte qui collabore avec la police - est un autre descendant de cette lignée. L'un des livres pour lesquels il est devenu célèbre est The Professional Thief by a Professional Thief (Chicago, University of Chicago Press, 1937). C'est l'autobiographie d'un voleur, d'un arnaqueur, appelé Chic Conwell dans le livre mais dont le véritable nom était Broadway Jones. Parmi les étudiants de Sutherland, il y avait des gens comme Alfred R. Lindesmith, qui a écrit un livre magnifique, Opiate Addictions (Bloomington, Principia Press, 1947) qui a fait date. L’idée était que le crime est juste une autre sorte de comportement humain, de comportement social. Et donc nous l'étudions de la même manière que nous aurions pu étudier les tribunaux, les universités, les usines. Juste un autre spécimen de la vie sociale humaine.

Au même moment, à l'université, certains criminologues essayaient de prédire qui violerait les conditions mises à la libération conditionnelle 2. J'ai appris très tôt certaines choses à ce propos. L'un de mes amis, qui travaillait comme psychologue dans une prison en Illinois, m'avait rapporté un fait très intéressant. Il m’avait dit : «Nous faisons passer des tests psychologiques à tous les condamnés pour voir ceux qui sont trop fous pour pouvoir sortir. Tu

2. Howard Becker fait vraisemblablement référence à certains travaux menés ou dirigés par Ernest Burgess, professeur de sociologie à l'Université de Chicago, travaux qui visaient à « rationaliser " la prise de décision en matière de libération conditionnelle ( parole ») au moyen d'échelles de prédiction du risque de récidive (Burgess E., "Is Prediction Feasible in Social Work? An inquiry Based upont a Sociological Study of Parole Records", Social Forces, 1928, 7, pp. 533-545 ; Bruce A.A., Burgess E.W., Harno A.M., "A Study of the Indeterminate Sentence and Parole in the State of Illinois", Journal of the American institute of Criminal Law and Criminology, 1928, 19-1 ; Laune F.F., Predicting Criminality: Forecasting Behavior on Parole, Northwestern University in the Social Sciences, 1, Evansoton, Northwestern University. Dans les sources secondaires, voir Harcourt B.E., Against Prediction. Profiling, Policing, and Punishing in an Actuarial Age, Chicago/London, The University of Chicago Press, 2006, pp. 47-76. 
ne le croiras peut-être pas, mais $98 \%$ de nos condamnés sont déments ». J'avais répondu : «Comment expliques-tu cela ? ». Et il m’avait répondu : "C'est simple, nous avons peur de leur donner de bons résultats aux tests... Que se passerait-il si nous disions qu'ils sont sains d'esprit, qu'ils sortaient, et qu'ils commettaient un horrible crime ? Nous serions vraiment en difficulté. Le plus sûr est donc de dire qu'ils sont fous, pour que personne ne les laisse sortir au prétexte que nous avons dit qu'ils étaient sains d'esprit. »

Donc, très tôt, j'avais été immunisé, j'avais appris à ne pas croire trop en la criminologie académique. J'avais été immunisé aussi en grandissant à Chicago. Je ne pense pas que la ville était réellement plus corrompue que d'autres villes américaines à l'époque - mais Chicago était corrompue et tout le monde le savait. Quand je travaillais comme musicien dans des bars, je voyais la police entrer dans le night-club, le club de striptease, et je voyais le propriétaire du club lui donner un billet de 100 \$. Et je savais pourquoi il le donnait. Donc j'avais conscience - la conscience d'un citoyen ordinaire - que ce que faisait la police n'était pas ce qu'elle était supposée faire.

La chose toute entière était basée sur des fausses prémisses et des fausses données, parce qu'on savait aussi très bien que la police mentait toujours sur les chiffres. Il y a un épisode célèbre à ce propos à Chicago. Les primes d'assurance pour les entreprises - pour les vols et choses de ce genre - dépendent du nombre de crimes qui sont enregistrés : plus ils sont nombreux, plus le coût de l'assurance est élevé. Comme payer plus n'est pas du goût des entreprises, les pressions exercées sur la police pour qu'elle n'enregistre pas trop de crimes sont importantes. Cela a été jusqu'au point où la police tenait deux types de registres : le premier pour les compagnies d'assurance, et le second pour savoir ce qu'ils faisaient. La disparité entre les deux devint si grande qu'à la fin, les compagnies d'assurance dirent : "Là, c'est ridicule ». Il y a eu un grand nettoyage, on fit venir le président de l'École de criminologie de l'Université de Californie - un officier de police très fameux, également criminologue -, lequel dit ceci aux gens de Chicago : «Si nous commençons à tenir des registres honnêtes, on va avoir une vague de crimes, une grosse. Êtes-vous prêts à ça ? » Ils n'avaient pas le choix, donc c'est ce qui s'est passé...

L'un de mes livres favoris, parce qu'il a fait voler en éclats tout un pan de la pensée conventionnelle, est un livre de Jack D. Douglas intitulé The Social Meanings of Suicide (Princeton, Princeton University Press, 1967). Ce qu'il prenait pour cible était, en réalité, toute la tradition durkheimienne. Le suicide de Durkheim est le modèle auquel se réfèrent ceux qui se demandent comment faire de la recherche à partir de statistiques générales sur les populations. L'une des grandes découvertes de Durkheim était l'importance de la communauté pour que le taux de suicide reste bas. Il expliquait le taux peu élevé de suicides parmi les catholiques par le fait qu'ils formaient davantage une communauté que les protestants et les juifs. Douglas a examiné comment ces chif- 
fres étaient construits et a découvert ce qui, sans doute, était évident pour tout le monde. Aux États-Unis - où il a mené son enquête - il existe une grande différence entre les juridictions où la cause de la mort est établie par le coroner du lieu, qui est un officier élu, et ceux où elle est déterminée par un médecin légiste, qui est un professionnel. Dans les districts catholiques, le coroner, élu, n'avait pas envie d'incommoder les familles en disant que quelqu'un s'était suicidé, ce qui signifiait qu'il ne pourrait pas être enterré dans le cimetière de l'église. Donc il ne le disait pas! Quand des arrondissements de ce type passèrent du coroner élu au médecin professionnel, le taux de suicide augmenta... C'est un résultat très important : comme la branche la plus positiviste de la criminologie dépendait de ce type de chiffres, ce fut un argument puissant pendant de nombreuses années.

Edwin Sutherland a également fait beaucoup pour les démythifier. Dans White Collar Crime (New York, Holt, Rinehart \& Winston 1949), il écrit : «Prenons A\&P - Atlantic \& Pacific Company, une grande chaîne d'épiceries, avec des magasins partout. Régulièrement, ils sont accusés de fausser les pesées. Aux clients, ils facturent une livre de viande - seize onces, mais vous n'en avez reçu que quatorze. C'est une infraction à la loi pénale, mais ça n'apparaît jamais dans les statistiques criminelles. Pourquoi pas ? En justice, ce n'est pas poursuivi comme une activité criminelle, c'est poursuivi au civil parce qu'ils veulent que ça s'arrête, ils veulent obtenir une injonction, ils veulent obtenir des dédommagements pour toutes les factures faussées. Mais donc, comme ils en font des affaires civiles, cela n’est jamais enregistré comme délit, et n'apparaît jamais comme tel. » Et il ajoute : "Si ces activités étaient poursuivies au pénal, comme des délits, qu'adviendrait-il de la fameuse corrélation entre - par exemple - le fait de venir d'un famille désunie et d'avoir des parents divorcés, et celui de commettre des délits ? Les dirigeants d'A\&P, de l'Atlantic \& Pacific Company, ne viennent pas d'une famille désunie ! ». Qu'ont fait les criminologues professionnels de ce puissant argument ? Fondamentalement, ils l'ont l'ignoré : «Mieux vaut ne pas y penser. On ne peut rien y faire, la qualité des statistiques est assez bonne ainsi ».

Quand je suis entré en sociologie, ces deux divisions étaient bien connues. Une partie des gens faisait essentiellement de la sociologie et étudiaient ce type d'activités. L'autre partie travaillait essentiellement pour la police et le système pénal : ils établissaient des corrélations entre telle ou telle variable et la délinquance ou la criminalité. Ils calculaient quels condamnés allaient violer leur liberté conditionnelle. Les deux tendances étaient représentées dans les universités, mais dans les universités davantage orientées vers la recherche, c'était l'approche sociologique qui dominait. D'autres universités formaient plutôt des jeunes gens à devenir surveillants de prison, agents de probation, etc. Vous obteniez un diplôme en criminologie et vous pouviez trouver un travail. Il y avait une Société Américaine de Criminologie, mais je n'ai même jamais su qui en était membre ; certaines des personnes que je connaissais l'étaient, sûre- 
ment - mais les gens que je connaissais travaillaient tous en sociologie, et leur public était fait de sociologues. Outsiders les a mis au défi parce qu'ils voulaient encore connaître les causes du crime, mais qu'il y avait de fortes chances qu'ils les trouvent là où rien ne peut être fait : si la pauvreté est une cause de la criminalité, alors la criminalité existera toujours, parce qu'il existera toujours de pauvres gens.

Avant d'écrire Outsiders, j'avais écrit deux articles sur l'usage de marijuana ; j'y avais fait quelque chose que je savais provocateur, qui était de procéder à un changement de mots, très simple. Je n'avais pas parlé d' "addiction à la marijuana », je n'avais pas parlé d' «abus de marijuana », mais d' " usage de marijuana » [en anglais « use » au lieu de « abuse » NDLR]. Les gens qui comprennent les nuances ont réagi : «Oh, qu'est-ce que c'est que ça ? » J'avais ôté la connotation négative : vous utilisez de la marijuana comme vous utilisez $\mathrm{du}$ shampoing, c'est la même chose. Et c'est de cette façon que j'ai mené mon étude : la loi pénale n'y entre en scène qu'une seule fois, quand je dis qu'apprendre à utiliser de la drogue pour le plaisir consiste pour partie à apprendre comment éviter d'avoir des ennuis avec la police. Ce n'est pas difficile à apprendre, mais il faut l'apprendre, même si, pour les gens que j'ai interviewés, la police - la possibilité d'être arrêté - était insignifiante. Pour le savoir conventionnel, ces articles étaient un défi : les drogues étaient habituellement regardées comme une solution à des problèmes personnels, même par les médecins et les biologistes (vous êtes névrosé, vous êtes énervé, vous prenez des drogues pour vous calmer). L'idée que quelqu'un puisse devenir accro à l'hérö̈ne pour éviter des problèmes est, en fait, ridicule : une fois que vous êtes accro, là, vous avez réellement des problèmes ! Et ce qui est certainement vrai, c'est que la plupart des gens (apparemment tous les gens) qui faisaient ce genre de travail n'avaient pas d'expérience personnelle ni avec les drogues, ni avec les personnes accros à la drogue, ni avec les usagers de drogues. Personnellement, j'avais l'expérience de la marijuana : j'en fumais moi-même et je savais que c'était une sorte de drogue inoffensive, bien plus inoffensive que l'alcool. D’un autre côté, j'avais travaillé avec un musicien qui était accro à l'héroïne, et je savais que ce n'était pas une partie de plaisir. Un exemple : je jouais dans un trio - moi, un saxophoniste et un batteur. Le batteur était un très bon batteur, mais c'était un junkie. Et régulièrement, 9 heures sonnaient, nous étions censés commencer à jouer et... «Où est-il ? » Il était debout, à un coin de rue, quelque part, à attendre quelqu'un qui allait venir avec de la drogue. C'est pour cela qu'il arrivait en retard.

Donc, je savais certaines choses à ce propos. Et tout ce discours - que les gens utilisent des drogues pour résoudre leurs problèmes - me semblait absurde. Mais en écrivant ces papiers, je n'avais pas prouvé que les gens n'essayaient pas de résoudre leurs problèmes avec des drogues. Je savais que ce n'était pas vrai, mais ma recherche ne le prouvait pas. Je n'avais pas mesuré leurs troubles de personnalité, et il est évident que je n’aurais pas pu résoudre 
la question de cette façon. Et quinze ans ou vingt ans plus tard, j'ai réalisé que ce n'était pas ce qui était en jeu. Ce qui était en jeu, c'est comment les gens apprennent à interpréter ce qu'ils éprouvent intérieurement, leurs expériences physiques et psychologiques. Comment savent-ils qu'ils planent ? Comment savent-ils qu'ils sont malades ? Ce n'est pas évident. Vous n'apprenez pas ce genre de choses quand vous êtes enfant, mais vous les apprenez. Tout le monde ne reconnaît pas les mêmes symptômes. Il y a toute une branche d'anthropologie médicale qui porte exclusivement sur cela ; puis, plus tard, la sociologie médicale. C'est donc à ce domaine de recherche que mon travail appartient, mais je ne le savais pas...

$C E C$ : Quand vous dites qu'il y avait une branche plus sociologique et une branche plus criminologique, est-ce que finalement la criminologie est autre chose qu'une science liée au fonctionnement concret d'institutions de contrôle? C'est-à-dire de gens qui feront la critique de la sociologie, en disant, "vous voulez expliquer, mais nous on veut arrêter la délinquance. C'est très bien ce que vous dites, mais il y a de la délinquance : comment est-ce qu'on fait? "

H.B. : La réponse la plus simple, c'est de dire qu'ils n'ont jamais rien fait à propos du problème du crime. Et je pense que c'est généralement vrai pour tous les sociologues qui prétendent résoudre des problèmes sociaux. C'est assez facile de voir quel est le problème et ce qui le cause, mais ce n'est pas facile de changer ces choses. J'en ai fait personnellement l'expérience, à un niveau beaucoup plus spécifique, quand mes collègues et moi étudiions une école de médecine. Nous y avons passé trois ans. Nous ne faisions pas ce que les étudiants faisaient, mais nous étions avec eux pendant qu'ils soignaient les patients, travaillaient au bloc opératoire, etc. À la fin de notre recherche, presqu'aucun professeur n'était intéressé par ce que nous avions trouvé, mais huit ou dix membres de la faculté ont lu ce que nous avions écrit. Ils ont été déçus : nous n'avions pas de recommandations, nous ne disions pas ce qu'ils devraient faire. Je leur ai dit : « Je n'ai pas de recommandations, je ne suis pas professeur dans une école de médecine. Mais si vous me dites ce que vous voudriez changer, je peux peut-être vous donner quelques idées. » Ils me répondirent : «Eh bien, par exemple, lorsque nous faisons passer les examens, nous savons que les étudiants mémorisent des pages et des pages ; ils mémorisent toute cette matière, ils nous la restituent pendant l'examen, et puis ils l'oublient. Et c'est quelque chose que nous n'aimons pas. » Donc je leur ai dit : «Quel type d'examen donnez-vous ? » Ils m'ont répondu : «Le genre habituel, vous savez... Il y a une question, écrivez la réponse en une heure ». Je leur ai dit: "Supposons que vous donniez un autre genre d'examen. Chaque étudiant a deux patients à examiner, doit faire leur histoire médicale, prescrire le travail de laboratoire, faire un diagnostic, suggérer un plan de traitement, tout ça; ensuite, vous examinez les mêmes patients et vous évaluez ce qu'il a fait ». Ils nous ont regardés : «Ce serait difficile ». Je leur ai dit : «Pourquoi ? Cela semble simple». «Eh bien, cela va prendre beaucoup de temps! Vous ne 
comprenez pas, nous sommes très occupés. Nous avons nos recherches, nous avons nos propres patients, nous avons notre travail administratif, nous n’avons pas de temps pour ça ». Je leur ai dit : «Eh bien, c'est la solution » (rires). Si vous en trouvez une qui soit meilleure, félicitations ! Et c'est généralement le cas : c'est facile de voir comment une masse de problèmes peuvent être résolus, mais les solutions évidentes nuisent aux intérêts de quelqu'un. Vous avez vos propres intérêts professionnels. Dans une prison, par exemple, le directeur, les surveillants, toutes les organisations ont leurs privilèges, leurs coutumes, leurs traditions, et savent comment s'en arranger. Et vous qui passez par là, vous leur diriez « débarrassez-vous de tout cela »? "Ce que vous recommandez n'est pas bon pour moi, donc je n'en veux pas ». À moins de parvenir à trouver le moyen de satisfaire tous les intérêts, c'est très difficile de changer. Si vous essayez de changer, cela devient juste une chose de plus avec laquelle ils doivent composer - eux, les gens qui sont le problème. Et ce que les gens qui mènent ces opérations ne comprennent jamais, c'est qu'ils font partie du problème parce qu'ils ont leurs propres intérêts à protéger. J'ai donc décidé que la sociologie était dépourvue de sens pratique. J'ai eu un jour une expérience magnifique au Canada. Le gouvernement canadien avait installé une Commission sur l'utilisation non médicale des drogues. Le président était un professeur de droit, un homme très distingué, le doyen de la Faculté de droit de McGill. Il nous avait conviés un jour, Ed Schur de New York, Terry Morris de Londres et moi pour discuter de ce problème. À la fin de la journée, il nous dit: "Tous les trois, vous continuez à me dire que la seule manière de se débarrasser du problème de la marijuana est de légaliser son utilisation ». Et nous de répondre : «Oui, c'est cela » (c'était intéressant, parce que Terry Morris était une personne beaucoup plus conservatrice que Schur ou moi ; or, il avait dit : « oui, pratiquement, c'est la seule façon d'y arriver»). Sur quoi, le président, endossant, en quelque sorte, le rôle de représentant du gouvernement de Sa Majesté, nous dit : «Messieurs, je vous ai exposé que le peuple canadien ne veut pas légaliser la marijuana, il veut en être débarrassé ». À quoi je répondis : «d'accord, je vais vous dire comment vous en débarrasser ». Ils venaient juste d'en découdre avec le Mouvement Québécois, dont une frange violente faisait exploser les boîtes aux lettres, etc. Ils avaient adopté quelques lois drastiques pour régler le problème, lois qu'ils avaient récemment suspendues. Je lui dis : "Reprenez ces lois, arrêtez les gens au hasard dans la rue, fouillez-les. Si vous trouvez de la drogue, abattez-les. Fouillez les maisons au hasard. Si vous trouvez de la drogue, brûlez-les ». Il dit : «Êtes-vous en train de me dire que rien d'autre ne marchera? » Je lui répondis : «Oui » et je pense qu'en réalité, c'est vrai (rires).

CEC : Le managérialisme est en train de devenir un courant dominant, y compris dans le champ pénal. Il repose sur le discours de Law \& Economics, développé à l'Université de Chicago par les économistes et les juristes qui, dans le sillage de Gary Becker, pensent le droit à partir de la microéconomie. A priori, on est loin de la criminologie : dans son introduction aux Essays in the 
Economics of Crime and Punishment (New York, Columbia University Press, 1974), Gary Becker indique qu'il veut produire, à propos des comportements qui enfreignent la loi pénale, une théorie qui ne soit pas criminologique. La criminalité est conçue comme une activité qui permettrait à un individu de maximiser son utilité, mais qui génèrerait des externalités négatives pour la communauté. La pénalité a donc pour objectif de limiter ces externalités négatives par l'action sur trois variables - la probabilité d'arrestation, la probabilité de condamnation et la sévérité de la peine. L'interface entre le gouvernement et l'individu étant la fiction de l'homo oconomicus, le problème posé est un problème de décision. L'individu doit décider quel temps et quelles ressources il va allouer à des activités légales et illégales, compte tenu des opportunités et $d u$ risque pénal lié aux activités illégales. Le gouvernement doit décider quelles ressources policières, judiciaires et pénitentiaires allouer pour donner force à la loi; mais ces ressources étant rares, il doit choisir quelle quantité d'infractions et d'infracteurs impunis il doit accepter. Outsiders a été une bombe par rapport à la criminologie positiviste. Mais, dans la mesure où les nouvelles politiques criminelles sont fondées sur le discours de Law and Economics, dans la mesure aussi où elles n'entendent plus agir sur les causes sociales du crime mais se limitent à agir sur trois variables pénales à partir de considérations managériales, la critique semble désormais devoir se déplacer. Comment pensez-vous que l'on puisse résister à ce type de discours?

H.B. : Je pense que c'est la même chose. Le piège, quand on s'occupe de managérialisme, c'est l'économie : ce sont les coûts et les bénéfices. Donc, le problème, c'est comment vous établissez les coûts et les bénéfices. Par exemple, quel est le coût de l'utilisation de la marijuana ? Quels sont les bénéfices ? Personne ne prend jamais en compte les bénéfices pour les consommateurs, leur plaisir. Personne ne traite réellement la question comme on le ferait en affaires. J'ai eu, il y a plusieurs années, un étudiant qui a fait une thèse exceptionnelle sur le business de l'héroïne à San Antonio au Texas. Il venait de San Antonio, il avait été à l'école là-bas, il connaissait tous les vendeurs de drogues parce qu'il avait été à l'école avec eux. Donc, il est retourné à San Antonio. Il a pris les têtes de chapitre d'un manuel de marketing, et il les a interviewés comme s'ils faisaient tourner une affaire ordinaire. "Combien payez-vous pour les matières brutes ? Combien par kilo d'héroïne ? »Question intéressante : l'emballage. La question qu'il a posée était vraiment une question merveilleuse : «Combien d'onces vous faites avec un kilo ? " Ce devrait être un nombre standard, mais habituellement ils « coupent » l'héroïne pour avoir plus d'onces à partir d'un seul kilo, en fonction de ce qu'ils y ajoutent. Il leur a aussi demandé : "Comment faites-vous votre publicité ? » Parce que si vous vendez quelque chose, les gens doivent savoir que vous le faites, et où ils peuvent vous trouver pour acheter. Comment vous y prenez-vous ? Et combien cela coûte-t-il ? Combien devez-vous payer à celles et ceux qui travaillent pour vous - quelles sont vos charges salariales ? Et combien de personnes employez-vous ? La découverte la plus intéressante était que les dealers qui 
engagent des membres de leur famille, comme les fils de leurs sœurs, et les associent à l'affaire, finissent ruinés parce qu'ils ont des dépenses qui excèdent ce qu'ils pourraient s'offrir et qu'ils perdent de l'argent. Les gens qui ont moins pitié de leur famille disent : "Non, je suis désolé, tes garçons ne sont pas bons, je ne les engage pas ». Et ils font bien. Donc, si vous devez traiter ces questions réellement en termes économiques et mettre un prix sur chaque chose, et ensuite avoir un bon argument pour tel prix, je suppose que cela mettrait un terme à bien des discussions.

Je pense que la chose principale, s'agissant du managérialisme, c'est leur utilisation de mesures dénuées de sens et de pertinence. Parce qu'ils mesurent tout ce qu'ils pensent pouvoir mesurer et ignorent le reste qu'ils traitent comme futile, pas important. De même, ils ignorent les défauts bien connus des données du type de celles sur lesquelles ils se basent. Il y a, vraiment, une littérature assez considérable sur les erreurs des données quantitatives. Vous connaissez mon livre favori sur ce sujet ? C'est un livre d'Oskar Morgenstern, On Accuracy of Economic Observations (Princeton, Princeton University Press, 1950). Morgenstern fut très important en économie à cause de la théorie des jeux - l'un des grands jalons, vous savez. Mais il travaillait aussi dans le champ du commerce extérieur, du commerce entre pays, et savait que beaucoup de nombres utilisés dans ce champ sont très imprécis. Il a pris une marchandise qui est soigneusement mesurée : l'or. Chaque pays publie des chiffres chaque année sur la quantité d'or qu'ils ont exportée à différents endroits et la quantité qu'ils ont importée. Par exemple, vous êtes en Allemagne, vous publiez des chiffres : "Nous avons exporté telle quantité en France ». Et la France publie des chiffres : "Nous avons importé telle quantité d'Allemagne ». Si vous comparez les deux nombres, ils devraient être identiques. Morgenstern a fait cette comparaison pour un grand nombre de paires de pays limitrophes, de sorte que le problème du temps de transport ne se posait pas. Quel est le rapport entre ces deux nombres, qui devraient être identiques ? De temps en temps, très rarement, c'était 1 . Plus souvent, c'était 2, 3, 4, 10, 50 - et ce n'était pas des comparaisons entre le Zaïre et l'Islande ! Il en a conclu qu'à l'évidence, ce chiffre n'est pas fiable. Et puis il a trouvé d'autres erreurs grossières : ce merveilleux article qui avait découvert, dans les données du recensement américain, un grand nombre de veuves adolescentes dans une réserve indienne Navajo - des veuves de 13 ans, comment cela se pouvait-il ? Une erreur de codage : quelqu'un avait confondu la colonne du statut marital et la colonne de l'âge. Tout le monde sait qu'il y a des erreurs de codage, disait Morgenstern, et tous les textes publiés devraient examinés en détail pour repérer ce type d'erreurs. Le recensement n'est pas différent. Si vous travaillez avec des nombres à une telle échelle, vous avez un tas d'erreurs.

CE्E : Si on développe cet argument, le danger est peut-être qu'ils disent que c'est une question d'efficacité. Qu'il suffit de produire de meilleures données. 
H.B. : Oui, mais c'est un bon argument. Prouvez-moi que vos données sont bonnes! La charge de la preuve est renversée, parce que nous voyons non seulement qu'il y a des problèmes, mais que ces problèmes sont endémiques. Morgenstern a fait ses calculs pour l'or pour un grand nombre de pays et un grand nombre d'années, et il y a toujours de sérieuses erreurs ; les statistiques policières sont toujours manipulées, etc. Bien sûr, ils répondront ce que vous dites. Quoi que vous disiez, ils répondront quelque chose. Mais à l'occasion, vous les attrapez, comme Sutherland les a attrapés quand il a montré que la décision de traiter des faits constitutifs d'infractions au pénal ou au civil était une source majeure d'erreurs dans les données. Une source majeure, non pas d'erreurs, mais de choix. C'est un choix des managers.

CEC : Revenons à la thèse de votre étudiant. Si l'on se met dans la peau d'un agent du système de justice pénale, et qu'on raisonne en termes de Law \& Economics on pourrait très bien dire: "Cette personne tire des bénéfices importants de son commerce. Il faut que donc affecter à cette activité un risque pénal égal ou supérieur aux bénéfices qu'elle peut en retirer. " En quoi ce type d'études donne-t-il des arguments suffisants pour contrer le managérialisme?

H.B. : Le managérialisme n'a pas fait son entrée dans le monde des affaires parce qu'il était logique ou qu'il était réceptif à un certain type d'argument logique. Le managérialisme, c'est une façon d'étudier à la mode... C'est le manager de telle société qui dit «Oh, on devrait le faire aussi »- toutes les décisions se prennent comme ça. Et puis, périodiquement, ce qui était rationnel change, la stratégie change, non pas parce que l'autre s'est avérée fausse, mais parce que c'est ce que fait telle autre personne. Mon père a travaillé dans la publicité toute sa vie durant. Il traitait avec ces gens et disait qu'ils étaient les plus illogiques de la terre, qu'aucun amoncellement de faits n'y changeait rien, jamais. Donc vous ne pouvez vraiment pas les changer, même si vous avez de bons arguments. Il y a un livre de Melville Dalton que j'aime beaucoup, Men Who Manage (New York, John Wiley, 1959). Dalton était un étudiant d'Everett Hughes, comme moi. Il a travaillé entre huit et dix ans au Personnel (aujourd'hui on dirait « Ressources humaines ») dans une série de très grandes firmes aux États-Unis, en rédigeant des notes de terrain tout au long. S'agissant du crime, il avait une merveilleuse théorie. Les employés volent toujours. C'est une partie des frais fixes, qui est habituellement évaluée à $5 \%$ du chiffre d'affaires. Mais, disait-il, c'est une erreur de penser à cela en termes de «vols ». La manière la plus appropriée de penser le phénomène, c'est d'y voir un système de gratification informelle. Il donnait des exemples. Mon favori concerne le vice-président d'une grande entreprise, qui avait une maison de campagne et qui voulait y construire une volière pour des oiseaux. Il y avait des charpentiers qui travaillaient pour l'usine. Plusieurs d'entre eux sont venus chez lui, ont apporté du bois et des matériaux de l'usine - tout cela, payé par la société -, pour lui construire cette volière. C'était complètement illégal. Non seulement c'était illégal, mais c'était ridicule pour la société. 
Pourquoi les employés l'ont-ils fait ? Parce que, dès lors, ils étaient autorisés à voler ce dont ils avaient besoin pour faire des réparations chez eux, pour les petits travaux qu'ils faisaient pour d'autres personnes, etc. Bien sûr, vous pourriez dire que c'est du vol, que tout le monde volait - mais ça n'explique rien. Ce qui explique la chose, c'est que le vice-président voulait sa volière, et qu'il a récompensé ceux qui la lui ont construite en les autorisant à voler. C'est un schéma très commun. Dalton avait beaucoup d'exemples, partout, dans toutes les sociétés où il avait travaillé. Finalement, le livre, au fond, traite de la politique intérieure des organisations, où les manières habituelles d'agir sont faites et défaites. Si vous voulez avoir un effet sur le vol, vous devez avoir un effet sur cette politique. Vous pourriez l'affecter de l'extérieur, c'est certain, si certains des managers de ces sociétés qui ont eu recours à ces procédés malhonnêtes étaient envoyés en prison pour dix ans : ce serait un message qu'ils reconnaîtraient.

Comment être débarrassé du managérialisme ? C'est comme cet homme au Canada qui me demandait comment être débarrassé de la marijuana. Vous les trouvez, vous les abattez... C'est très difficile de se débarrasser de ces choses parce que les causes - pas seulement les causes, mais les processus par lesquels elles ont été établies - existent depuis un bon moment maintenant. La manière dont je pense à tout cela, c'est que ces schémas managériaux sont une sorte d'utopie: "Si tous faisaient les choses exactement comme je dis qu'ils doivent les faire, alors, nous aurions tel résultat ». " $\mathrm{Si}$ » soulève une énorme question, parce que la réponse est sûrement qu'ils ne feront pas les choses comme cela, et alors quoi ? Comment voulez-vous interférer avec ça ? Aucun d'entre nous n'est en position d'interférer. Que pouvons-nous faire ? Écrire des livres ? Je pense qu'il y a un peu de mégalomanie chez nous, qui consiste à penser que nous pouvons avoir un effet important sur des tendances sociales majeures et sur les événements. Le sociologue qui a sans doute eu le plus grand effet aux États-Unis était Daniel Patrick Moynihan. C'était un sociologue qui avait toutes sortes d'idées sur l'éducation, la famille, les structures, la race, etc. Des idées folles, je pense, même si c'était quelqu'un de très intelligent. Il devint sénateur pour l'État de New York (1977-2001) et fut membre du cabinet de Richard Nixon (Urban Affairs). Il était mieux placé que qui que ce soit en sciences sociales (à l'exception des économistes) pour avoir un effet sur la politique. Qu'a-t-il accompli ? Rien... Les gens aiment beaucoup me dire que mon vieux collègue Erving Goffman est responsable de la libération des malades mentaux. Vous le savez : il a écrit Asiles et, d'une manière ou d'une autre, cela a permis que tous les malades mentaux soient libérés. Accessoirement, cela a produit un autre problème, celui dit des «sans domicile fixe ", parce que les gens que l'on a laissés quitter l'hôpital n'ont plus d'endroit où dormir. Évidemment, Goffman n'est pas responsable de cela. Il ne s'agit pas non plus de dire que Goffman n'a pas joué un rôle dans cette libération; mais il s'inscrit dans un lent mouvement de transformation de l'appréhension de ce type de problème. À côté des recherches académiques, il y a eu 
aussi des films, comme Vol au-dessus d'un nid de concous (1975) ou La fosse aux serpents (1948). Il y a eu des psychiatres comme Thomas S. Szasz, qui a écrit un grand livre intitule The Myth of Mental Illness: Foundations of a Theory of Personal Conduct (Sydney, Harper \& Collins Publishers, 1974) dans lequel il disait que la «maladie mentale »n'est pas une maladie. Les gens ont des difficultés à vivre, des problèmes dans leur vie, oui ; mais ils n'ont pas une maladie, même si nous continuons à traiter leurs problèmes comme une maladie. Beaucoup de gens, dans le monde entier, pensaient cela. Dans le même temps, Ronald Reagan est devenu gouverneur de l'État de Californie et il a cherché les moyens de faire des économies, parce que ce sont des institutions très coûteuses. Il a pris le risque d'incommoder tout un tas de personnes importantes, parce que chaque hôpital psychiatrique était une source de revenus pour la communauté. Dans la ville de Napa, dans la Californie rurale, la plus grosse industrie, c'est l'hôpital psychiatrique. Beaucoup de gens y travaillaient, l'hôpital achetait des fournitures, de la nourriture, tout ce qui se produit là-bas. Il employait des gens pour faire la lessive. Tout ça a été fermé. Mais si vous fermez, vous créez un grave problème économique pour la ville. Reagan a pris ce risque.

CEC : De la même manière qu'il y a eu une transformation lente de la manière de penser le problème psychiatrique, il semble qu'il existe un déplacement dans la manière de penser le problème de la pénalité. Et le changement s'est accompagné d'une augmentation énorme de la population détenue. En psychiatrie, on a sorti les gens des asiles; là, au contraire, on fait entrer les gens en prison.

H.B. : Le nombre de personnes en prison aux États-Unis a crû de manière aussi spectaculaire en grande partie à cause des lois qui exigent qu'à la troisième condamnation, on soit incarcéré pour une longue durée. Ça s'appelle «three strikes»- je me suis toujours demandé ce que les gens qui ne connaissent pas le baseball comprennent $\mathrm{du}$ «three strikes »... Les lois des «three strikes » ont été une sorte de mode que l'aile droite [des professionnels de la politique] a été capable d'exploiter. Le coût est énorme. Pour la peine de mort, même chose : des États essaient maintenant de s'en débarrasser tellement c'est cher. La constitution telle que les cours l'interprètent aujourd'hui exige que la personne condamnée à mort ait la meilleure défense possible ; et celle-ci coûte très cher. Et donc ces affaires se prolongent, encore et encore, de sorte qu'une personne peut être dans le couloir de la mort durant trente ans. Ce n'est pas une politique rationnelle, elle ne résout rien du tout. Mais elle génère du profit, et c'est la raison pour laquelle les petites villes se battent pour avoir une nouvelle prison construite à proximité. Je viens de lire un manuscrit, un livre qui peut-être sera publié, sur les processus politiques mis en ouvre pour obtenir qu'une prison soit construite dans votre ville, surtout dans les villes frappées par la délocalisation. "Les usines ont fermé, nous avons tous ces gens sans travail, tentons d'avoir une prison, c'est une bonne affaire ». C'est bon 
aussi pour les entreprises de construction : c'est un gros chantier pour quelqu'un qui travaille dans ce secteur et ça génère beaucoup d'emplois. La vraie cause de l'accroissement de la population des prisons est là. L'union des surveillants de prison - le syndicat - est désormais une force puissante dans la politique californienne. Ils disent : "Si nous réduisons les peines, mettons, d'un an - un an seulement, pas la vie -, nous aurons moins d'emplois, moins de surveillants et donc moins de membres ». Et comme les prisons sont parsemées partout sur le territoire, ils sont capables d'influencer les élections législatives dans de nombreuses circonscriptions. La cause de l'augmentation des taux de criminalité, c'est ça. La cause de la multiplication des prisons, c’est ça.

CEC : Durant les années 1960 et 1970, on est passé d'une analyse du crime en termes un peu étiologiques - le milieu social, les milieux populaires sont plus criminogènes que d'autres - à l'idée que les institutions ont un rôle. Aujourd'bui l'accent est plutôt mis sur la responsabilité individuelle. L'analyse criminologique dominante insiste sur la responsabilité de l'individu - son milieu social ne compte plus, sauf en termes de "facteur de risque " - et là on rejoint la question de la prédiction, la question de la dangerosité.

H.B. : Tout d'abord, juste de manière rhétorique, je dirais que c'est une idée intéressante. Appliquons-la à tous les criminels, à commencer par les cadres des entreprises, les cadres des banques, et voyons ce qu'ils font. Il y a pour le moment aux États-Unis trois ou quatre cadres auparavant très haut placés qui se trouvent en prison, et leurs peines, suivant les standards français, sont incroyables - dix ans, vingt ans, c'est sérieux. L'idée, bien sûr, est et a toujours été de faire un exemple et de vous montrer ce qui pourrait vous arriver si vous êtes pris. Mais la plupart des gens ne croient pas qu'ils vont être pris. Lorsque j'étais gosse, je fumais de la marijuana, tous mes amis du monde de la musique fumaient de la marijuana, un tas de gens fumaient de la marijuana. Nous étions tous, pour l'essentiel, de très gentils enfants, de bonne famille ; nous étions éduqués, nous étions à l'université. Pourquoi faisions-nous ça ? Nous étions sûrs de ne pas nous faire attraper. Pourquoi en étions-nous sûrs ? Parce que personne parmi nos connaissances n’avait jamais été attrapé. De temps à autre, il arrivait que quelqu'un soit pris. Nous avons toujours considéré que cette personne avait été trop loin. Vous ne pouvez pas passer devant le commissariat de police en fumant un joint et espérer que rien ne se passe : c'est juste idiot. Mais si vous ne faites pas ça, il n'y a aucun souci à se faire. Je dirais que sans doute, ce qui explique le mieux pourquoi les gens font tant de ces choses qui les conduisent à être arrêtés est un mauvais calcul de probabilités : «Cela ne m'arrivera jamais ». Les pratiques s'enracinent dans certaines communautés pour de bonnes raisons. Ce n'est pas sans raison que les communautés noires aux États-Unis sont les lieux où on vend de la drogue : elles ne peuvent pas se défendre contre cela. Une communauté blanche de classe moyenne ne l'autoriserait pas. Simplement, ils iraient tout de suite chez le maire, lui diraient qu'ils vont mener campagne contre lui la prochaine fois, 
qu'il aura un opposant sérieux, et qu'il a intérêt à se tenir prêt ou à les débarrasser de ces gens. La communauté noire ne peut pas se défendre contre ce type de business. Donc vous y avez un business local. Ils engagent des gosses du coin, c'est le meilleur job dans les alentours, vous pouvez vous faire plus d'argent en faisant ça, vous pouvez avoir un job à proximité, votre cousin peut décrocher le boulot pour vous, etc. Alors, vous avez parlé de responsabilité individuelle. Lorsque les gens parlent de ça, ils imaginent quelqu'un qui vit dans un vide, où toutes les possibilités sont ouvertes et disponibles, de sorte que c'est une juste le choix individuel de la personne de devenir un mauvais garçon. Mais en réalité, les gens ne choisissent pas d'être un mauvais garçon, ils le deviennent. Je ne pense pas que vous puissiez avoir une discussion rationnelle avec les gens qui développent ces arguments, parce que théoriquement, ils sont trop engagés. Un autre livre que j'aime beaucoup est celui de Donald R. Cressey, Other People's Money: A Study in the Social Psychology of Embezzlement (Glencoe, The Free Press, 1953), dans lequel il étudie comment une personne cesse de sentir une responsabilité morale. Imaginez que travaillez dans une banque et vous que avez une mauvaise habitude : vous allez aux courses, vous pariez sur des chevaux. Jusqu'à présent cela ne vous a pas gagné, mais vous êtes sûr qu'un jour ce sera le cas. Entre temps, vous avez besoin d'argent, et vous êtes là, dans cette banque, entouré d'argent. Vous savez comment le voler puisque votre travail implique, justement, des mouvements de fonds. Comment justifiez-vous d'en voler ? Tout le monde sait que les banques sont criminelles et vous le savez mieux que tout le monde puisque vous y travaillez. Vous savez ce qu'elles font. Et donc c'est acceptable pour vous de voler. "Je ne suis pas une mauvaise personne, je le rendrai ». Et là, l'auditeur arrive et vous demande : «Où est l'argent ? »

CEC : Vous connaissez certainement le livre de Stanley Coben, Against Criminology (New Brunswick, NJ: Transaction Books, 1988). En exergue, il a écrit cette phrase: "Il faut appartenir à une tradition, pour pouvoir la baïr comme il faut (" to hate it properly "). Ce serait bien sûr ridicule de soutenir qu'il faut instituer la criminologie en France pour pouvoir la haïr correctement. Il reste qu'une anti-criminologie y est vraiment nécessaire. Une " anti-criminologie ", c'est-à-dire un travail de démystification, de critique d'un discours criminologisant. Ceci semble d'autant plus important que le "criminalisme" est en quelque sorte la forme nouvelle que prend le racisme, et que le crime ou la criminalisation sont, politiquement, des façons particulièrement dangereuses de justifier l'exclusion. La criminalisation permet de dépolitiser toute une série de questions sociales. Vous avez dit que la sociologie n'est pas pratique. Mais diriez-vous qu'elle n'est pas polémique? pas politique? Vous avez écrit un jour un papier intitule "De quel côté sommes-nous? " "Whose Side Are We On ", Social Problems, vol 14, 1967, pp. 239-247) Seriez-vous d'accord avec Pierre Bourdieu, qui décrivait en plaisantant la sociologie comme un sport de combat? Comment définiriez-vous ce pour quoi (ou ce contre quoi) vous vous battiez quand vous avez écrit Outsiders ou Whose Side Are We On? 
H.B. : Pour moi, ce n'est pas un sport de combat. La science, quand elle est bien faite, est et devrait être une entreprise coopérative. Je ne fais pas un fétiche de séparer mes vues politiques de mon travail sociologique, mais je ne pense pas qu'elles y aient une place importante. Il est probable que c'est dans le choix des sujets de recherche que leur influence est la plus importante, et dans mon insistance à prendre en compte toutes les personnes impliquées dans l'activité que j'étudie. Ceci implique pour une école, par exemple de considérer non seulement les élèves, mais aussi les professeurs, les administrateurs, les personnes qui assurent la maintenance des bâtiments, qui les nettoient et vident les poubelles. Si vous faites cela - c'est l'argument de «Whose Side Are We On ?»-, le plus souvent, vous finissez, pour le meilleur ou pour le pire, par être du côté des gens qui ont le moins de pouvoir. Pour la simple raison que les personnes qui en ont davantage ont souvent davantage de choses à cacher, dont elles peuvent être blâmées, dont elles peuvent être tenues pour responsables. Lorsque vous faites de la recherche comme je l'ai fait, vous trouvez presque toujours que les choses ne sont pas comme elles ont été décrites, ce qui signifie que vous défiez les descriptions qui protègent le pouvoir. Nul besoin pour cela d'être politiquement radical : il vous suffit d'être un chercheur honnête, qui fait un travail d'enquête minutieux. Cela vous engagera dans quelque conflit avec les gens au pouvoir, bien sûr; mais je ne pense pas que ce soit mon travail, comme sociologue, de faire ça. Vous imaginez bien que je ne me soucie pas trop d'ébranler les puissants et d'une certaine façon, j'aime ça. C'est bon pour eux, ça leur maintient les pieds sur terre, ça les rend un peu plus responsables. Mais mener des luttes politiques, ce n'est pas de la sociologie. Non que je veuille édicter une règle qui dirait que les sociologues ne devraient pas s'engager en politique. Loin de là ! Simplement, je trouve que ce n'est pas gratifiant, parce qu'il me semble que si peu a été accompli. Je pense que des changements se produisent, et que nous autres, les chercheurs y jouons un rôle. Mais je crois aussi que celui-ci est limité, comparé à celui des intérêts politiques, économiques ou autres. Le plus grand effet que nous ayons est probablement d'aider à changer le climat politique, comme par exemple celui relatif au mariage entre personnes du même sexe, qui s'est modifié après des années et des années de discussion et d'argumentation. Nous pouvons certainement prendre part à cela, comme Goffman quand il a écrit Asiles. Écrire quelque chose qui donnerait à voir les schémas managériaux comme des utopies aiderait à modifier le climat (quel qu'il soit) sur cette question, mais je n'attendrais pas que cela se produise très rapidement. Cela prendra peut-être cinquante ans ou cent ans. Et très probablement, à ce moment-là, quelque chose d'autre l'aura déjà détruit. Ces modes ont une très courte espérance de vie. 